F. S. Cater, Department of Mathematics, Portland State University, Portland, Oregon 97207, USA

\title{
AN ELEMENTARY PROOF THAT ANALYTIC FUNCTIONS ARE OPEN MAPPINGS
}

\begin{abstract}
We give an elementary real analysis proof that an analytic function on an open region, not constant on any non-void open subset, is an open mapping.
\end{abstract}

One of the standard theorems of complex analysis is that the range of a nowhere constant analytic function on an open region, is an open set. It follows that such a function is an open mapping, i.e., maps open sets to open sets. The usual proofs come from other complex analysis theorems that in turn depend on such matters as contour integrals, for example (see [2], and [3]). We will give a relatively short and elementary proof using real analysis. No inverse functions, derivatives or integrals of any kind are involved, and little calculation is required. In [1] Serge Lang gave a real analysis proof. However, it involved several issues and calculations that will not enter our argument.

The result should be evident from the following Lemma we hope is of some independent interest.

Lemma 1. Let $f$ be analytic on a neighborhood of the closed disc

$$
D=\{z:|z| \leq r\}
$$

and let $f$ be constant on no non-void open set. Let

$$
f(h)=f(0)+c_{j} h^{j}+\sum_{k=j+1}^{\infty} c_{k} h^{k} \quad \text { for } \quad|h| \leq r,
$$

Key Words: analytic function, open mapping, power series.

Mathematical Reviews subject classification: 30D10, 54C10.

Received by the editors June 6, 2001 
and let

$$
\sum_{k=j+1}^{\infty}\left|c_{k}\right| r^{k-j}<\left|c_{j}\right|
$$

Let $d$ denote the distance from the point $f(0)$ and the set $f(B)$, where

$$
B=\{z:|z|=r\} .
$$

Then $d>0$ and $\left\{w:|w-f(0)|<\frac{d}{2}\right\} \subset f(D)$.

Proof. From $(*)$ we get $\left|\sum_{k=j+1}^{\infty} c_{k} h^{k}\right|<\left|c_{j} h^{j}\right|$ for $|h|=r$, and $f(0)$ does not lie in the set $f(B)$. But $f(B)$ is compact because $B$ is compact and $f$ is continuous. So $d>0$. Let $T$ be a number with $|T-f(0)|<d / 2$. It suffices to prove that $T \in f(D)$. We proceed by contradiction. Assume that $T \notin f(D)$. Now $f(D)$ is also compact, so we can (and do) select $v \in D$ such that

$$
|T-f(v)|=\inf \{|T-f(z)|: z \in D\} .
$$

Note that $T$ is closer to $f(0)$ than to any point in $f(B)$. It follows that $f(v) \notin f(B), v \in D \backslash B$ and $|v|<r$. Let

$$
f(v+h)=f(v)+b_{s} h^{s}+\sum_{k=s+1}^{\infty} b_{k} h^{k} \quad\left(b_{s} \neq 0\right)
$$

for $h$ in some neighborhood of 0 . Choose a real number $p$ such that

$$
\begin{gathered}
0<p<r-|v|, \\
\sum_{k=s+1}^{\infty}\left|b_{k}\right| p^{k-s}<\left|b_{s}\right|, \\
\left|b_{s}\right| p^{s}<|T-f(v)| .
\end{gathered}
$$

Let $a_{1}$ and $a_{2}$ be the angles such that $b_{s}=\left|b_{s}\right|\left(\cos a_{1}+i \sin a_{1}\right)$, and

$$
T-f(v)=|T-f(v)|\left(\cos a_{2}+i \sin a_{2}\right),
$$

and let the angle $a_{3}$ satisfy

$$
s a_{3}+a_{1}=a_{2} .
$$


Put $H=p\left(\cos a_{3}+i \sin a_{2}\right)$. It follows from (4) that the complex numbers $b_{s} H^{s}$ and $T-f(v)$ have the same angle (are in the same direction), and by (3), $\left|b_{s} H^{s}\right|<|T-f(v)|$. It follows that

$$
|T-f(v)|-\left|b_{s} H^{s}\right|=\left|T-f(v)-b_{s} H^{s}\right|
$$

From (2) we obtain

$$
\left|\sum_{k=s+1}^{\infty} b_{k} H^{k}\right|<\left|b_{s} H^{s}\right| .
$$

From (5) and (6) we obtain

$$
\begin{aligned}
|T-f(v+H)| & =\left|T-f(v)-b_{s} H^{s}-\sum_{k=s+1}^{\infty} b_{k} H^{k}\right| \\
& \leq\left|T-f(v)-b_{s} H^{s}\right|+\left|\sum_{k=s+1}^{\infty} b_{k} H^{k}\right| \\
& =|T-f(v)|-\left|b_{s} H^{s}\right|+\left|\sum_{k=s+1}^{\infty} b_{k} H^{k}\right| \\
& <|T-f(v)| .
\end{aligned}
$$

Finally, $|v+H| \leq|v|+|H|=|v|+p \leq r$ by (1), so $v+H \in D$. This conflicts with the choice of $v$.

Now let $f$ be analytic and nowhere constant on an open region $U$. We prove that for $z \in U, f(z)$ is interior to $f(U)$. To this end, translate so that $z=0$, take the series expansion at 0 , and make $r$ small enough that $(*)$ holds as in Lemma 1. We leave the rest.

Remark. We can use this approach to prove the Fundamental Theorem of Algebra. Let $f$ be a non-constant polynomial. Let $\{z:|z| \leq M\}=A$ be a closed disc such that $|f(z)|>|f(0)|$ if $z \notin A$. Let $|f(v)|$ be minimal for $|f|$ on $A$. Then $|f(v)|$ is minimal for $|f|$ (v complex). If $|f(v)|>0$, use the technique in the proof of Lemma 1 to find $v+H$ with $f(v+H)$ closer to 0 than $f(v)$ is. Likewise we can give an elementary real analysis proof of the theorem:

Let $f$ be a complex function on $\mathbb{C}$ that is analytic at every point where $f$ does not have a pole. Let $f$ have a pole at infinity. Then $f(\mathbb{C})=\mathbb{C}$. 


\section{References}

[1] S. Lang, Complex Analysis, Addison-Wesley, Reading, 1977 (Chapter II, $\S 5$, Theorems 5.1, 5.2).

[2] N. Levinson and R. M. Redheffer, Complex Variables, Holden-Day, San Francisco, 1970 (Theorem 6.1, Chapter 5).

[3] R. Remmert, Theory of Complex Functions, Springer-Verlag, New York, 1991 ( 5 , Chapter 8). 